\title{
A Power-Efficient Ultra-Wideband Pulse Generator Based on Multiple PM-IM Conversions
}

\author{
Enbo Zhou, Xing Xu, King-Shan Lui, and Kenneth Kin-Yip Wong
}

\begin{abstract}
A novel photonic generation of power-efficient ultrawideband (UWB) pulse by incoherent summation of two asymmetric monocycle pulses with inverted polarities is experimentally demonstrated. The principles of multiple cross-phase modulations in a highly nonlinear fiber and multiple phase modulation to intensity modulation conversions in a commercially available arrayedwaveguide grating are used. The combined UWB pulse exploiting spectral efficiency of $\mathbf{5 0 . 5 9 \%}$ in experiment is fully compliant with the Federal Communications Commission (FCC) spectral mask without power attenuation. The FCC-compliant UWB pulse gains larger than 11.5- and 6-dB improvement against monocycle and doublet pulses after power attenuation to respect the FCC spectral mask, respectively. Such a power-efficient UWB pulse with pulse duration of approximately 320 ps has potential to achieve high-speed transmission without pulse overlapping and obvious distortion.
\end{abstract}

Index Terms-Microwave photonics, nonlinear signal processing, ultra-wideband (UWB)-over-fiber, wireless technology.

\section{INTRODUCTION}

I MPULSE radio-based ultra-wideband (UWB) technology exploiting extremely wideband spectrum attracts rapidly increased interests after the regulation specified by the U.S. Federal Communications Commission (FCC) of unlicensed use of spectral band from 3.1 to $10.6 \mathrm{GHz}$ with power density lower than $-41.3 \mathrm{dBm} / \mathrm{MHz}$ [1]. Such low spectral density with wide available spectral range results in a reasonable radiated signal power for indoor use, if the whole occupied spectrum fully complies with the FCC spectral mask.

To further address the incapability of penetration of walls with budget radiated signal power, UWB-over-fiber technology is suggested to interconnect the infrastructures in the electrical domain and the optical domain. Photonic generation of low-order derivative of Gaussian pulse, i.e. monocycle and doublet pulses with simplicity, has been widely reported in recent few years [2]-[10]. A well-known technology of phase modulation to intensity modulation (PM-IM) conversion by

Manuscript received January 21, 2010; revised March 25, 2010; accepted May 08, 2010. Date of publication May 24, 2010; date of current version June 25,2010 . This work was supported in part by grants from the Research Grants Council of the Hong Kong Special Administrative Region, China (projects HKU7179/08E and HKU7183/09E).

E. Zhou, X. Xu, and K.-S. Lui are with the Photonic Systems Research Laboratory, Department of Electrical and Electronic Engineering, The University of Hong Kong, Hong Kong, SAR, China (e-mail: nbzhou@eee.hku.hk; xuxing@eee.hku.hk; kslui@eee.hku.hk).

K. K.-Y. Wong is with the Photonic Systems Research Laboratory, Department of Electrical and Electronic Engineering, The University of Hong Kong, Hong Kong, SAR, China, on leave at the Massachusetts Institute of Technology, Cambridge, MA 02139 USA (e-mail: kywong@eee.hku.hk).

Color versions of one or more of the figures in this letter are available online at http://ieeexplore.ieee.org.

Digital Object Identifier 10.1109/LPT.2010.2050469 using a detuned frequency discriminator, i.e., a fiber Bragg grating (FBG)-based filter or a tunable bandpass filter (TBF) after phase-modulated signals has been reported extensively in recent years [2]-[4]. By coherent or incoherent summation of two polarity inverted pulses, monocycle and doublet pulses can be generated [5]-[10]. It is noticeable that those active devices-based schemes, i.e., semiconductor optical amplifiers (SOAs) and distribution-feedback (DFB) lasers, are negatively influenced by the slow carrier lifetime due to band-filling effect, even if a high-order UWB pulse is generated [11]. On the other hand, by tailoring the optical spectrum of an ultrashort pulse with a pulsewidth of the hundreds of femtoseconds level, arbitrary waveforms with FCC-compliant radio-frequency (RF) spectra can be achieved [12]. However, it is noted that the spectra of either monocycle or doublet pulses have distinguished frequency components in low-frequency band $(<2 \mathrm{GHz})$. Those frequency components violate the dip in FCC spectral mask for noninterference operation with other wireless technologies, especially in the global positioning system (GPS) band $(1.57542 \mathrm{GHz})$, where a suppression ratio of $34 \mathrm{~dB}$ comes into a challenge. As a result, power attenuation has to be implemented to respect the FCC spectral mask for those monocycle and doublet pulses, which sacrifice the spectral efficiency.

It is reported recently that the specially designed FBGs utilizing apodization technology can generate power-efficient UWB pulses fully compliant with FCC mask without any external RF source [13], [14]. Furthermore, design of such an FCC-compliant UWB pulse generator in consideration of spectral gain profile of UWB antenna has been demonstrated as well [15]. This approach is highly precise in pulse shaping and spectrum tailoring, and an integrated solution can be expected. However, the trade-off in system complexity may raise the operation cost. A power-efficient UWB pulse generator with simplicity is highly desirable for the UWB-over-fiber technology at the risk of slightly higher operation cost.

In this letter, a novel power-efficient UWB pulse generator based on a highly nonlinear fiber (HNLF), performing as a multiple-phase modulator, which combines with an arrayed-waveguide grating (AWG), performing as a multiple-frequency discriminator, is implemented. By incoherent summation of two asymmetric monocycle pulses with inverted polarities, the generated UWB pulse with high spectral efficiency of $50.59 \%$ fully compliant with the FCC spectral mask has been experimentally demonstrated. Furthermore, our approach has potential in adapting different modulation formats due to pulse position programmable and pulse polarity switchable when the two wavelengths are located in the opposite side of AWG channels. Also high-speed transmission can be achieved due to ultrafast response time of the HNLF inherited from its third-order susceptibility $\left(\chi^{(3)}\right)[8],[16]$. 


\section{PRINCIPLE}

The UWB waveforms with potential in efficiently exploiting the FCC spectral mask can be described as [14]

$$
p(t)=\sum_{n=0}^{L-1} w[n] g\left(t-n T_{0}\right)
$$

where $w[n]$ are $L$ weight coefficients, $T_{0}$ is the pulse spacing, and $g(t)$ is the Gaussian monocycle. It is investigated in [14] that the FCC-compliant UWB pulse without power attenuation needs $L$ is at least 7 . For lower order summation, $L=2,3$ for instance, the generated pulses are monocycle and doublet pulses which exploit low spectral efficiency. However, it is noted that this investigation is restricted to summation of symmetric monocycles. In the scheme of PM-IM conversion, a transient state leads pulse shape conversion to monocycle-like pulse when the optical carrier located at a linear slope of the transmission function of a detuned filter. Furthermore, if the optical carrier detuned to either the maximum or the minimum transmission point, i.e., the two edges, of the linear slope, an asymmetric monocycle pulse with equal upper and lower pulsewidth but adjustable proportion of absolute amplitude can be achieved. Such asymmetric monocycle pulse has even more undesirable frequency components in low frequency domain. However, by incoherent summation of a pair of asymmetric monocycle pulses with opposite polarity and appropriate time delay, an FCC-compliant UWB pulse with temporal waveform fitting to (1) for $L=7$ can be achieved. This is the first experimental demonstration, to the best of our knowledge, of the FCC-compliant UWB pulse, which requires summation of only two asymmetric monocycle pulses.

\section{EXPERIMENT}

The experimental setup for FCC-compliant UWB pulse generation is shown in Fig. 1. The pump light emitting at $1548 \mathrm{~nm}$ was first modulated by an intensity modulator (IM) to Gaussian pulse train. The IM was synchronized by a $12.5-\mathrm{GHz}$ clock source from Agilent N4906B bit-error-rate tester (BERT). The pattern was defined as one mark in every 125 bits, resulting in an equivalent repetition rate of $100-\mathrm{MHz}$ and pulsewidth [full-width at half-maximum (FWHM)] of 80 ps. An erbium-doped fiber amplifier (EDFA) after the IM was used to amplify the pump power to $8.74 \mathrm{dBm}$. Two probe light were emitted from two tunable laser sources (TLSs) around ITU WDM wavelengths of $191.4 \mathrm{THz}(1566.31 \mathrm{~nm})$ and $191.6 \mathrm{THz}$ $(1564.68 \mathrm{~nm})$ with a power of $2 \mathrm{dBm}$, respectively. The wavelengths for the two probe light will be specified in details in the following experiment for the generation of different UWB pulses. The two probe light were coupled by a 50:50 coupler and then amplified by an EDFA to $17.92 \mathrm{dBm}$ to compensate 8-dB loss in the AWG. A WDM coupler (WDMC) was used to couple the pump light and the two-probe light. The port for pump light with passing through wavelength from 1546.9 to $1549 \mathrm{~nm}$ can be also performed as a filter to block the amplified spontaneous emission (ASE) noise. The coupled three lights copropagated along a 400-m HNLF with zero-dispersion wavelength (ZDW) of $1554 \mathrm{~nm}$ and $\gamma \approx 14 \mathrm{~W}^{-1} \cdot \mathrm{km}^{-1}$. A commercially available AWG with channel starting wavelength of $191.4 \mathrm{THz}, 3-\mathrm{dB}$ bandwidth of $40 \mathrm{GHz}$, and channel spacing of $50 \mathrm{GHz}$ after the HNLF was used to perform as a

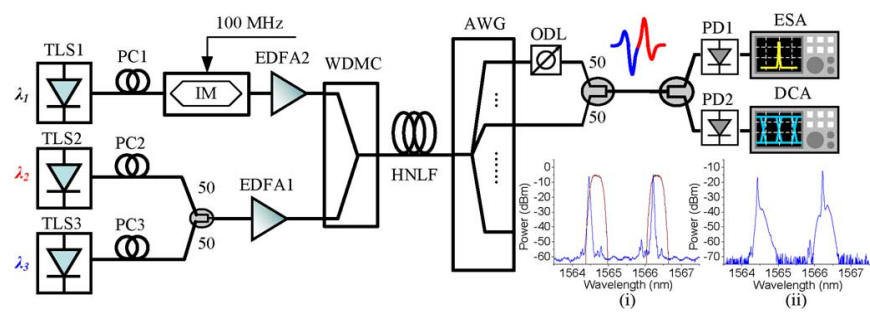

Fig. 1. Experimental setup for FCC-compliant pulse generation.

multiple-frequency discriminator for two probe light and block the pump pulse simultaneously.

The probe wavelengths locating at different positions of the transmission function of the AWG channel led to recovery of opposite polarities of asymmetric monocycle pulses. In our case, channel 1 (191.4 THz) and channel 5 (191.6 THz) were used due to dense channel spacing of the AWG available. In any case, adjacent channels should be avoided to reduce channel interference, especially for detuning operation. An optical delay line (ODL) was cascaded in channel 1 to obtain one pulse duration delay, which was approximately 160 ps. A 50:50 coupler recombined the two probe light from the two channels.

In order to compare UWB monocycle and doublet generation for comparison with the FCC-compliant UWB pulse, a similar experimental setup was used without major rearrangement. For monocycle pulse generation, only one probe wavelength and the corresponding channel of the AWG were used. The probe wavelength located in the linear slope of the transmission function of the AWG channel 1 was selected. Additionally, the ODL was removed due to single probe light involved. In order to avoid spectral overflow induced by short pulse, a $9.5-\mathrm{GHz}$ repetition clock resulting in a pulsewidth of approximately 105 ps with the same pattern defined previously was used. For generation of doublet pulse, even less modification to the experimental setup was required, as shown in Fig. 1. It only requires adjusting the two probe wavelengths to the opposite linear slope of the transmission function of the corresponding AWG channel, and the time delay to be approximately 80 ps occupying a half pulse duration time.

A digital communication analyzer (DCA), Agilent infiniium DCA 86100A, and an electrical spectrum analyzer (ESA), Agilent E4440A, were employed to measure the waveforms and spectra of the generated UWB pulses after two photodiodes (PDs), respectively. For UWB monocycle pulse generation, the wavelength of the probe light was $1566.162 \mathrm{~nm}$ located at the linear slope of AWG channel 5. The pulse waveform and the corresponding electrical spectrum are shown in Fig. 2(a) and (d), respectively. Additionally, two probe wavelengths were measured at 1566.162 and $1564.861 \mathrm{~nm}$, located at the linear slopes of AWG channels 5 and 1, respectively, for doublet generation. The corresponding waveform and electrical spectrum are shown in Fig. 2(b) and (e), respectively. After optimization on two probe wavelengths towards designed spectrum, the FCC-compliant UWB pulse shape and spectrum had been experimentally achieved, as shown in Fig. 2(c) and (f), respectively. A well-matched spectra between design and experimental measurement were observed. The slight deviation of the spectral components in low frequency domain $(<1 \mathrm{GHz})$ was mainly due to the minor fluctuation of the UWB pulse tail originated from the pump pulse. However, it still scrupulously respected 


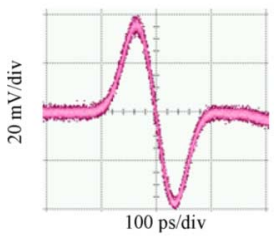

(a)

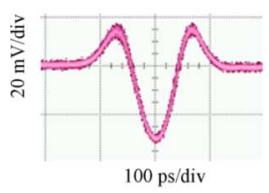

(b)

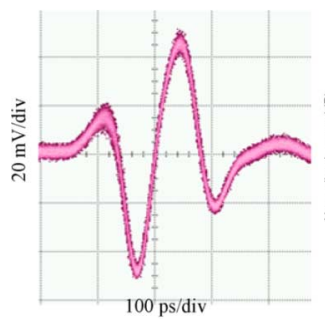

(c)

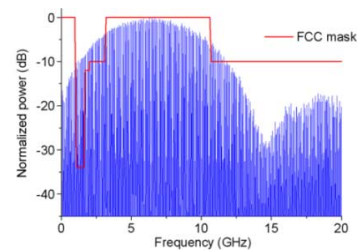

(d)

(e)

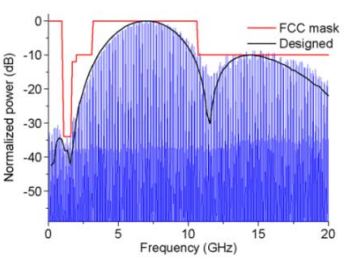

(f)

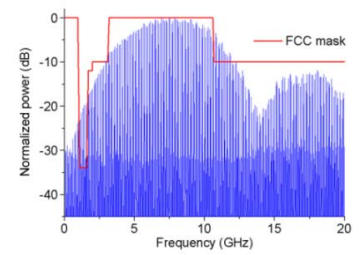

Fig. 2. Measured (a)-(c) waveforms and (d)-(f) the corresponding electrical spectra of the monocycle, doublet, and FCC-compliant pulses, respectively.

the FCC spectral mask. The measured spectral efficiency within the 3.1-10.6-GHz band was approximately $50.97 \%$. The probe light with optimized wavelengths of 1566.223 and $1564.461 \mathrm{~nm}$ were measured by an optical spectrum analyzer (OSA). The two wavelengths before and after the corresponding two channels filtering are shown in inset (i) and (ii) of Fig. 1, respectively. Being distinguishing from [3] and [4], the probe wavelengths here are located in the two edges, rather than the center, of the linear slopes, which are critical to achieve asymmetric monocycle pulses. The wavelength selection for generation of different UWB waveforms is summarized in Table I. The wavelength detuning of the two carriers relative to the corresponding AWG channel center $\left(\Delta \lambda_{i}, i=1,2\right)$ further indicates that the linear regions are approximately $0.1 \mathrm{~nm}$ in length and with center of $\pm 0.15 \mathrm{~nm}$ distant from the transmission peak. According to the electrical spectra measured in Fig. 2(d)-(f), compulsory electrical power attenuations on the monocycle and doublet pulses required to respect the FCC spectral mask are calculated to be 24.9 and $14.6 \mathrm{~dB}$, respectively. The calculated optical power of our FCC-compliant pulse gains larger than 11.5- and 6 -dB improvement against monocycle and doublet pulses, respectively.

\section{CONCLUSION}

A novel power-efficient UWB pulse generator based on multiple cross-phase modulations and PM-IM conversions in an HNLF and a commercially available AWG is experimentally demonstrated. The generated UWB pulse with spectral efficiency of $50.59 \%$ fully complies with FCC spectral mask without power attenuation. Larger than 11.5- and 6-dB improvement on optical signal power against monocycle and doublet pulses are achieved, respectively. Furthermore, the generated FCC-compliant UWB pulse benefiting from the ul-
TABLE I

WAVELENGTH SELECTION FOR DIFFERENT UWB WAVEFORMS

\begin{tabular}{ccccc}
\hline \hline UWB waveforms & $\lambda_{1}$ & $\lambda_{2}$ & $\Delta \lambda_{1}$ & $\Delta \lambda_{2}$ \\
\hline Monocycle & $1566.162 \mathrm{~nm}$ & & $0.148 \mathrm{~nm}$ & \\
Doublet & $1566.162 \mathrm{~nm}$ & $1564.861 \mathrm{~nm}$ & $0.148 \mathrm{~nm}$ & $-0.181 \mathrm{~nm}$ \\
FCC-compliant & $1566.223 \mathrm{~nm}$ & $1564.461 \mathrm{~nm}$ & $0.087 \mathrm{~nm}$ & $0.219 \mathrm{~nm}$ \\
\hline \hline
\end{tabular}

trafast response of HNLF with pulse duration of approximately 320 ps has potential to achieve high-speed transmission without pulse overlapping and obvious distortion.

\section{ACKNOWLEDGMENT}

The authors would like to acknowledge Sumitomo Electric Industries for providing the HNLF.

\section{REFERENCES}

[1] First Report and Order, (Revision of Part 15 of the Commissions Rules Regarding Ultra-Wideband Transmission Systems) FCC, Apr. 2002.

[2] J. Yao, F. Zeng, and Q. Wang, "Photonic generation of ultrawideband signals," J. Lightw. Technol., vol. 25, no. 11, pp. 3219-3235, Nov. 2007.

[3] F. Zeng, Q. Wang, and J. Yao, “All-optical UWB impulse generation based on cross-phase modulation and frequency discrimination," Electron. Lett., vol. 43, no. 2, pp. 121-122, Jan. 2007.

[4] Y. Dai and J. Yao, "Optical generation of binary phase-coded direct-sequence UWB signals using a multichannel chirped fiber Bragg grating," J. Lightw. Technol., vol. 26, no. 15, pp. 2513-2520, Aug. 1, 2008.

[5] Q. Wang, F. Zeng, S. Blais, and J. Yao, "Optical ultrawideband monocycle pulse generation based on cross-gain modulation in a semiconductor optical amplifier," Opt. Lett., vol. 31, pp. 3083-3085, 2006.

[6] J. Dong, X. Zhang, J. Xu, D. Huang, S. Fu, and P. Shum, "Ultrawideband monocycle generation using cross-phase modulation in a semiconductor optical amplifier," Opt. Lett., vol. 32, pp. 1223-1225, 2007.

[7] H. Chen, M. Chen, T. Wang, M. Li, and S. Xie, "Methods for ultrawideband pulse generation based on optical cross-polarization modulation," J. Lightw. Technol., vol. 26, no. 15, pp. 2492-2499, Aug. 1, 2008.

[8] J. Li, B. P. P. Kuo, and K. K. Y. Wong, "Ultra-wideband pulse generation based on cross-gain modulation in fiber optical parametric amplifier," IEEE Photon. Technol. Lett., vol. 21, no. 4, pp. 212-214, Feb. 15, 2009.

[9] E. Zhou, X. Yu, X. Zhang, W. Xue, Y. Yu, J. Mørk, and I. T. Monroy, "Photonic generation of ultrawideband monocycle and doublet pulses by using a semiconductor-optical-amplifier-based wavelength converter," Opt. Lett., vol. 34, pp. 1336-1338, 2009

[10] J. Li, S. Fu, K. Xu, J. Wu, J. Lin, M. Tang, and P. Shum, "Photonic ultrawideband monocycle pulse generation using a single electro-optic modulator," Opt. Lett., vol. 33, pp. 288-290, 2008.

[11] X. Yu, T. B. Gibbon, and I. T. Monroy, "Experimental demonstration of all-optical 781.25-Mb/s binary phase-coded UWB signal generation and transmission," IEEE Photon. Technol. Lett., vol. 21, no. 17, pp. 1235-1237, Sep. 1, 2009.

[12] J. D. McKinney, I. S. Lin, and A. M. Weiner, "Shaping the power spectrum of ultra-wideband radio-frequency signals," IEEE Trans. Microw. Theory Tech., vol. 54, no. 12, pt. 1, pp. 4247-4255, Dec. 2006.

[13] M. Abtahi, M. Mirshafiei, J. Magne, L. A. Rusch, and S. LaRochelle, "Ultra-wideband waveform generator based on optical pulse-shaping and FBG tuning," IEEE Photon. Technol. Lett., vol. 20, no. 2, pp. 135-137, Jan. 15, 2008.

[14] M. Abtahi, J. Magne, M. Mirshafiei, L. A. Rusch, and S. LaRochelle, "Generation of power-efficient FCC-compliant UWB waveforms using FBGs: Analysis and experiment," J. Lightw. Technol., vol. 26, no. 5, pp. 628-635, Mar. 1, 2008.

[15] M. Abtahi, M. Mirshafiei, S. LaRochelle, and L. A. Rusch, "All-optical 500-Mb/s UWB transceiver: An experimental demonstration," $J$. Lightw. Technol., vol. 26, no. 15, pp. 2795-2802, Aug. 1, 2008.

[16] J. Li, Y. Liang, and K. K. Y. Wong, "Millimeter-wave UWB signal generation via frequency up-conversion using fiber optical parametric amplifier," IEEE Photon. Technol. Lett., vol. 21, no. 17, pp. 1172-1174, Sep. 1, 2009. 